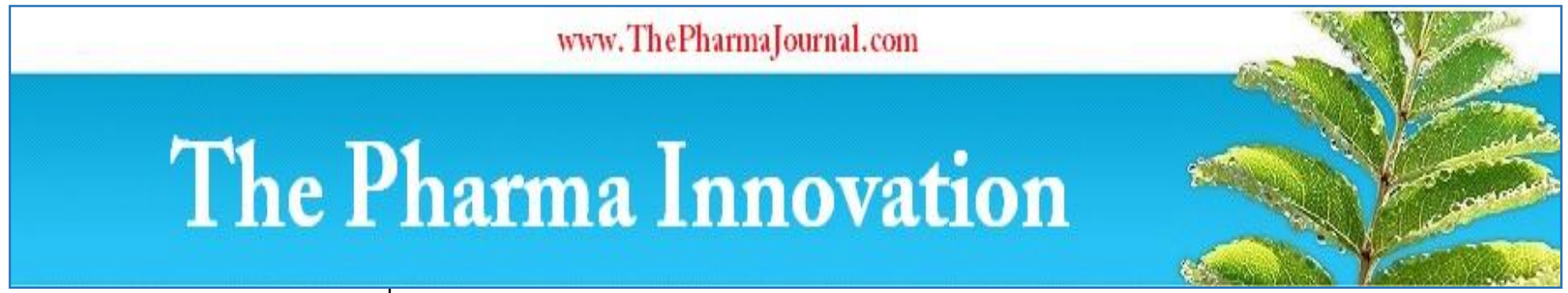

ISSN (E): 2277 - 7695

ISSN (P): 2349-8242

NAAS Rating: $\mathbf{5 . 0 3}$

TPI 2020; 9(6): 243-246

(C) $2020 \mathrm{TPI}$

www.thepharmajournal.com

Received: 10-04-2020

Accepted: 12-05-2020

MV Swamy Babu

PG student, Department of

Veterinary Surgery and

Radiology College of Veterinary

Science, Tirupati. Andhra

Pradesh, India

\section{P Veena}

Professor, Department of

Veterinary Surgery and

Radiology College of Veterinary

Science, Tirupati. Andhra

Pradesh, India

\section{RV Suresh Kumar}

Professor and University Head,

Department of Veterinary

Surgery and Radiology College of

Veterinary Science, Tirupati.

Andhra Pradesh, India

\section{P Amaravathi}

Assistant Professor, Department of Veterinary Pathology College of Veterinary Science, Tirupati.

Andhra Pradesh, India

\section{G Vani}

Assistant Professor, Department of Veterinary Surgery and

Radiology College of Veterinary

Science, Tirupati. Andhra

Pradesh, India
Corresponding Author:

\section{$P$ Veena}

Professor, Department of

Veterinary Surgery and

Radiology College of Veterinary

Science, Tirupati. Andhra

Pradesh, India

\section{Clinical, pathological and immunohistochemical bovine papillomatosis}

\author{
MV Swamy Babu, P Veena, RV Suresh Kumar, P Amaravathi and G \\ Vani
}

DOI: https://doi.org/10.22271/tpi.2020.v9.i6d.4775

\begin{abstract}
The present clinical study was conducted to study incidence, clinical symptoms, histopathological and immunohistochemical findings of bovine papillomatosis. Among total of 3812 cattle presented to the clinic, 52 cattle were having papillomas. No incidence of papillomas was recorded in buffaloes. Highest incidence of bovine papillomas was observed in cattle of 3 years age. Jersey crossbred cows were the most commonly affected breed followed by HF cross breed and non - descript cows. Most of the papillomas were located on teats only $(40.38 \%)$ followed by teats and udder (21.15\%). Histopathologically, fibro papilloma (60\%) was found to be most common papilloma followed by Angio keratotic acanthomatous papilloma (40\%). Immunohistochemical studies were conducted to know the rate of proliferation of bovine papillomas. Immunopositive reaction was observed against VEGF in Angio keratotic acanthomatous papilloma. EGFR and Ki67 immunopositivity was observed in fibropapillomas.
\end{abstract}

Keywords: Bovine papillomatosis, histopathological and immunohistochemical.

\section{Introduction}

Bovine papillomatosis is a contagious disease caused by bovine papilloma virus and characterized by the presence of warts or papilloma of various sizes on the skin, either localized to the teat or generalized (Somvanshi, 2011) ${ }^{[11]}$. Papilloma viruses (PVs) are small, non enveloped, double stranded DNA viruses with circular genomes replicate intranuclearly in infected cells. Thirteen types of the bovine papillomaviruses (BPVs) have been characterized in cattle; namely BPV1 to BPV13, which are divided into three genera, as Delta papilloma virus (BPV 1-2 and 13), Epsilon papilloma virus (BPV5 and 8) and Xipapilloma virus (BPV 3, $4,6,9,10,11 \& 12$ ) (Jeremiah et al., 2016) ${ }^{[5]}$.

In the bovine species, cutaneous papillomatosis is more common than in any other domestic animals. The disease affects young animals more often and more severely, but may also affect cattle of all age (Borku et al., 2007) ${ }^{[2]}$. The spread of the disease is usually by direct contact, many factors such as contaminated food and equipment, castration, injections, inheritance, nutritional imbalance, hormonal imbalance and suppressing the immune system may play roles in the spread of the disease. The aim of the present study was to describe the clinical, macroscopic, histopathological and immunohistochemical findings of bovine cutaneous papillomatosis.

\section{Materials and methods}

A total of 52 cattle aged between 1 to 8 years were involved in this study. Incidence of papillomas in bovines among a total number of bovine cases was recorded over a period of one year. The data regarding the incidence of bovine papillomas were collected and variables like age, breed, sex, location of papillomas and clinical symptoms were also recorded and analyzed. Diagnosis was done based on the clinical signs and histopathological findings.

\section{Histopathological studies}

Representative tissue samples were collected under local anaesthesia for histopathological studies. These samples were fixed in $10 \%$ buffered formalin and were processed routinely for histopathological examination. The sections of 5 micron thickness from embedded paraffin block were made and stained with Haematoxylin\& Eosin (H\&E). The sections were examined 
microscopically for histological changes (Culling, 1974) ${ }^{[3]}$.

\section{Immunohistochemical studies}

Paraffin sections of papillomas tissue samples were cut at 3 $4 \mu \mathrm{m}$ thickness mounted on APES (Amino Propyl Ethoxy Sialine) coated slides and incubated overnight at $37^{\circ} \mathrm{C}$. These slides were subjected to immunohistochemistry protocol.

\section{Procedure}

- Sections were deparaffinised by passing through xylene 2 changes 15 minutes each and dipped in absolute alcohol 2 changes to remove xylene. Washed under running tap water for $10 \mathrm{~min}$. Rinsed in distilled water for $5 \mathrm{~min}$.

- Kept in Tris EDTA buffer (pH-9.0) and hot water bath treatment was given for $20 \mathrm{~min}$ at $100^{\circ} \mathrm{C}$ to retrieve the antigenic sites.

- Cooled to the room temperature, kept in the distilled water $5 \mathrm{~min}$ and in Tris buffer saline for $5 \mathrm{~min}$.

- The slides were kept in the humid chamber and in the peroxidase block solution (3\% hydrogen peroxidase freshly prepared) for $30 \mathrm{~min}$ to block the endogenous peroxidase.

- Washed in Tris buffer saline for 3 times 5 min each. The power block solution using $1.5 \%$ BSA was poured on the tissue sections and kept for $30 \mathrm{~min}$.

- Primary antibodies (VEGF, EGF and Ki67) were added on the sections and slides were kept at room temperature for $2 \mathrm{hrs}$ or at $4^{\circ} \mathrm{C}$ over night.

- Washed in Tris buffer saline for 5 min each in 3 changes.

- Secondary antibody with HRP was added and kept for 1 hr. Washed in Tris buffer saline for $5 \mathrm{~min}$ each in 3 changes.

- Using Diaminobenzidine (DAB), the working coloring reagent was prepared by adding one drop of DAB to $1 \mathrm{ml}$ of substrate. The sections were kept in the coloring reagent for $30 \mathrm{~min}$.

- Washed in Tris buffer saline for 2 times and in tap water for $10 \mathrm{~min}$. Stained with Harris haematoxylin for $1 \mathrm{~min}$. Washed in tap water for 5 min. Then air dried and mounted with DPX.

- Result: Development of brown colour in the cells indicates positive reaction whereas blue colour development is due to staining with haematoxylin.

\section{Results}

Data pertaining to age, breed, sex, location of papillomas and clinical symptoms were shown in Table 1 to 5 .

Table 1: Incidence of bovine papillomatosis in different age groups of cattle $(n=52)$

\begin{tabular}{|c|c|c|c|}
\hline S. No & Age group & No. of animals & Percentage \% \\
\hline 1 & Above 3 yrs & 36 & 69.23 \\
\hline 2 & $1-3$ yrs & 14 & 26.92 \\
\hline 3 & Below 1 yr & 2 & 03.84 \\
\hline & Total & 52 & 99.99 \\
\hline
\end{tabular}

Table 2: Incidence of bovine papillomatosis in different breeds of cows $(\mathrm{n}=52)$

\begin{tabular}{|c|c|c|c|}
\hline S. No & Breed & No. of animals & Percentage \% \\
\hline 1 & Jersey cross bred & 27 & 51.92 \\
\hline 2 & HF Cross bred & 24 & 46.15 \\
\hline 3 & ND cows & 1 & 01.92 \\
\hline & Total & 52 & 99.99 \\
\hline
\end{tabular}

Table 3: Location of papillomas in bovine papillomatosis $(n=52)$

\begin{tabular}{|c|c|c|c|}
\hline S. No & Location of papillomas & No. of animals & Percentage \\
\hline 1 & Teats & 21 & 40.38 \\
\hline 2 & Teats \& udder & 11 & 21.15 \\
\hline 3 & Head \& neck & 6 & 11.53 \\
\hline 4 & Limbs (fore and hind) & 6 & 11.53 \\
\hline 5 & Generalized & 4 & 7.69 \\
\hline 6 & Eyes \& eyelids & 2 & 3.84 \\
\hline 7 & Ventral abdomen & 1 & 1.92 \\
\hline 8 & Tail base & 1 & 1.92 \\
\hline
\end{tabular}

Table 4: Table showing type of papillomas $(n=52)$

\begin{tabular}{|c|c|c|c|}
\hline S. No & Type of papillomas & No. of animals & Percentage \\
\hline 1 & Flat & 20 & 38.4 \\
\hline 2 & Filliform & 15 & 28.8 \\
\hline 3 & Cauliflower & 10 & 19.2 \\
\hline 4 & Flat, filliform and cauliflower & 5 & 9.61 \\
\hline 5 & Flat and cauliflower & 1 & 1.92 \\
\hline 6 & Flat and filliform & 1 & 1.92 \\
\hline
\end{tabular}

Table 5: Surface wise analysis of papillomas $(n=52)$

\begin{tabular}{|c|c|c|c|}
\hline S.NO & Surface of papillomas & No. of animals & Percentage \\
\hline 1 & Rough & 29 & $55.76 \%$ \\
\hline 2 & Smooth & 19 & $36.53 \%$ \\
\hline 3 & Rough and Smooth & 4 & $7.69 \%$ \\
\hline
\end{tabular}

Macroscopically, papillomas present on body parts other than teat and or udder were mostly of pedunculated type, where as papillomas on teat and udder were mostly sessile, non pedunculated and multiple in nature, and size ranging from $0.5 \mathrm{~cm}$ to $1 \mathrm{~cm}$. Grey and brown colored papillomas were most commonly found $(48.07 \%)$ followed by black $(28.84 \%)$, white $(21.15 \%)$ and black and brown (1.92\%). Most commonly observed papillomas were having rough surface $(55.76 \%)$ followed by smooth surface $(36.53 \%)$ and a combination of both rough and smooth surface $(7.69 \%)$.

In the present study, fibropapilloma $(60 \%)$ was found to be the most common papilloma followed by Angio keratotic acanthomatous papilloma (40\%).

Histologically, fibropapilloma sections revealed thickened epidermis, hyperkeratosis with irregular rete ridges extended down wards deep into dermis. In between there was huge proliferation of connective tissue in between rete ridges and in dermis. Hyperplastic stratum spinosum and granular layer were observed (Fig 1\&2).

Microscopically, Angio keratotic acanthomatous papillomas sections revealed hyperkeratosis with rete ridges of variable length was noticed within the dermis. There were variable sized dilated thin walled blood vessels and in few vessels well demarcated endothelial cells were observed. In two cases, there was prominent hemorrhage in dermis as well as in stratum corneum. Blood vessels were surrounded by fibrous tissue (Fig 3\&4).

Immunohistochemical studies against VEGF, EGFR and Ki67 were carried out in all the cases. Immunopositive reaction was observed against VEGF in the cytoplasm of endothelial cells of blood vessels and the basal layer of epidermis in angiokeratotic acanthomatous papilloma cases (Fig 5). Immunopositive reaction was noticed in different layers of epidermis in the cytoplasm of fibroblasts present in dermis against EGFR in fibropapillomas (Fig 6). Mild immunopositive reaction was noticed against Ki67 in the nuclei of basal layer of epidermis in few cases of fibropapillomas (Fig 7). 


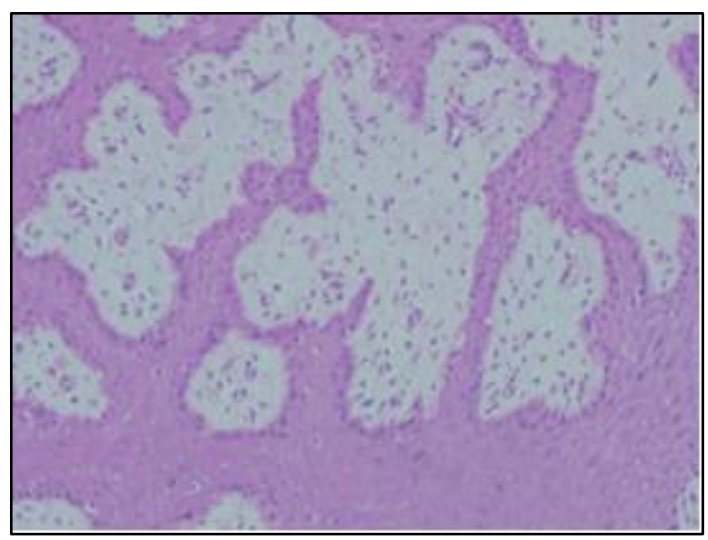

Fig 1: Showing fibropapilloma: different shapes of rete ridges. H\&E: $\mathrm{x} 40$

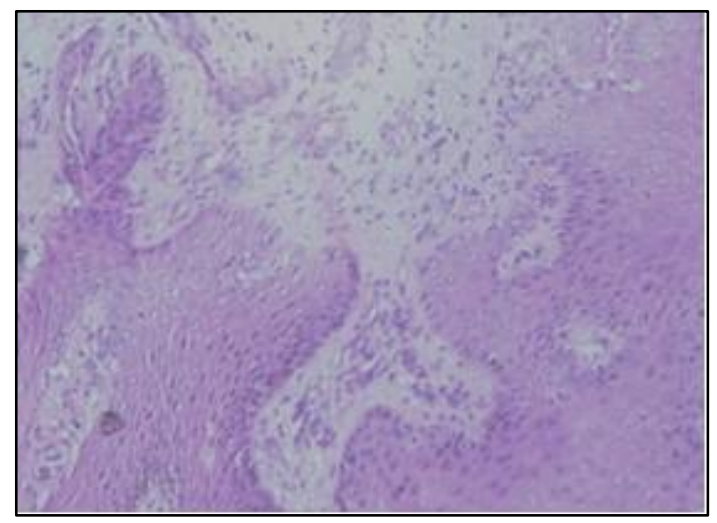

Fig 2: Fibropapilloma: showing hyperplastic cells in epidermis and presence of fibroblasts in the dermis. H\&E: x400

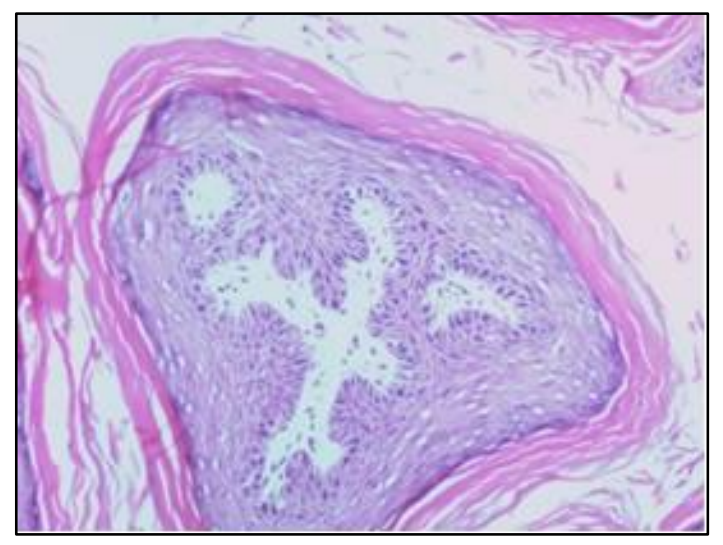

Fig 3: Showing angiokeratotic acanthomatous papilloma: Hyperkeratosis of dermis. \& E: x40

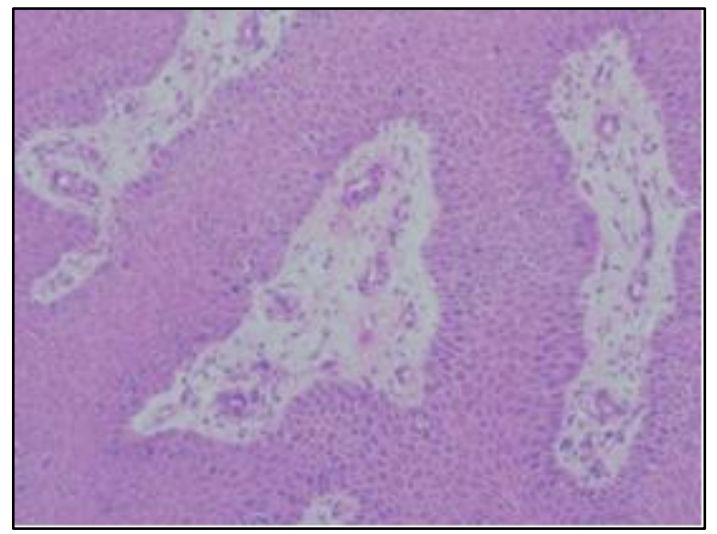

Fig 4 Showing angiokeratotic acanthomatous papilloma: Section showing fibrous tissue around blood vessels. H\&E: x100

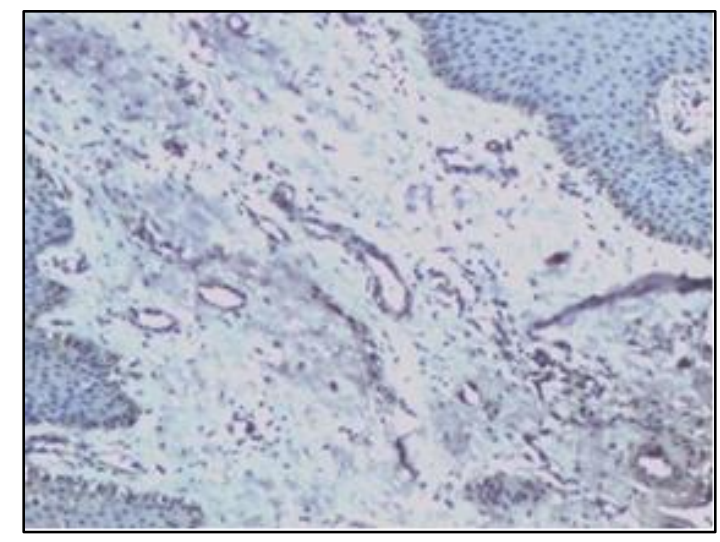

Fig 5: Note immunopositive reaction in the cytoplasm of endothelial cells of blood vessels and basal layer of epidermis. VEGF x100

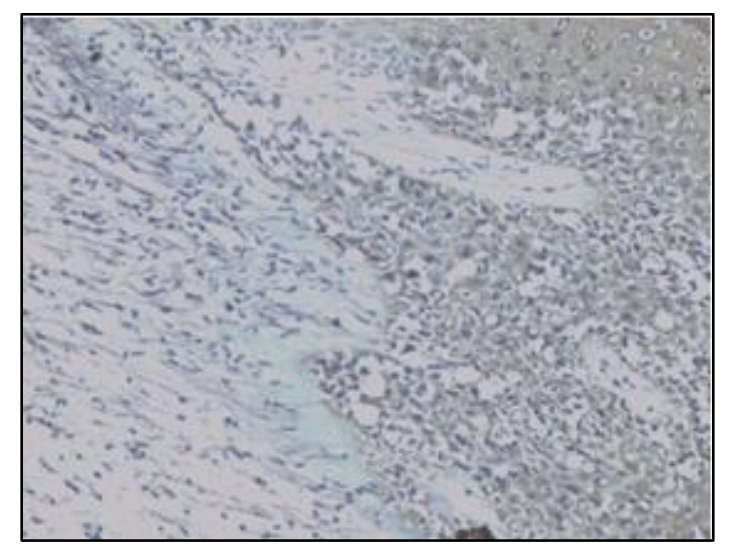

Fig 6: Note immunopositive reaction in cytoplasm of different layers of epidermis EGFR: $\mathrm{x} 400$

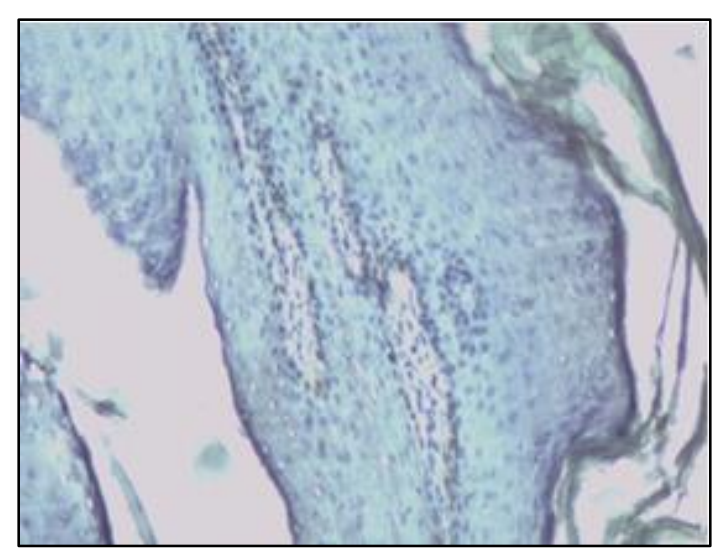

Fig 7: Note: mild immunopositive reaction in the nuclei of cells in basal layer of epidermis Ki67: x400

\section{Discussion}

The incidence of bovine papillomatosis was found to be high in age group of above 3 years followed by 1-3 years and was in agreement with the study of Hamad et al. (2018) ${ }^{[4]}$. The correlations in the breed wise incidence of bovine papillomatosis revealed highest incidence in Jersey cross bred cows followed by Holstein Friesian cross bred and Non descript cows. Ozsoy et al. (2011) ${ }^{[10]}$ reported highest incidence of bovine papillomas in Holstein cattle. However, the availability of different breeds in a particular geographical location would predispose certain breeds to get affected. The incidence was higher in cross bred animals. The incidence of bovine papillomatosis was found to be high in females. All the cases were recorded in female cattle only. Hamad et al. 
(2018) ${ }^{[4]}$ who also observed highest incidence of papillomas in female cattle. The reason might be the female cattle usually under stress factors such as gestation, lactation and progression in age. In the present study, maximum number of animals were having papillomas on teats only, followed by warts on udder and teats, concurring with the findings of Jelinek and Tachezy (2005) ${ }^{[6]}$, Mathi et al. (2016) ${ }^{[9]}$ and Hamad et al. (2018) ${ }^{[4]}$ who observed papillomas on teats and udder in mature cattle.

Confirmatory diagnosis of warts was achieved by histopathological examination of wart lesions. Histologically, fibropapillomas and angiokeratotic acanthomatous papillomas were the different types of papillomas diagnosed in the present study. The findings of the present study were in agreement with the observations of Tozato et al. (2013) ${ }^{[12]}$, Hamad et al. (2018) ${ }^{[4]}$ and Ugochukwu et al. (2018) ${ }^{[13]}$ who reported histological appearance of bovine papillomas as fibropapillomas and angiokeratotic acanthomatous papillomas.

VEGF is a potent angiogenic factor, produced by a variety of cell types, including keratinocytes, endothelial cells, macrophages, mast cells, and fibroblasts and is involved in several types of tumors, where it has been shown to influence both tumor neovascularisation and dissemination (Martano et $a l ., 2018)^{[8]}$. In the present study, by immunohistochemistry, expression of VEGF in the cytoplasm of endothelial cells of blood vessels and the basal layer of epidermis in angiokeratotic acanthomatous papilloma cases was observed, suggesting a possible role in papilloma development through angiogenesis (Martano et al., 2018) ${ }^{[8]}$.

Epidermal growth factor receptor (EGFR) is a key factor in epithelial malignancies, and its activity enhances tumor growth, invasion, and metastasis. EGFR plays an important role in maintaining normal cell function, dysregulation of EGFR signaling pathways contributes to the development of malignancy via effects on cell- cycle progression, inhibition of apoptosis, induction of angiogenesis, and promotion of tumor-cell motility and metastasis. In our study, EGFR Immunopositive reaction was noticed in different layers of epidermis in the cytoplasm of fibroblasts present in dermis which established that dysregulation of EGFR receptor signaling pathway is associated with the development and progression of malignancy.

Bovine papillomatosis can also be confirmed with immunohistochemical examination of the basal layer of the affected epithelial and connective tissue using antibodies against specific antigen of BPV1 and Ki67 protein (Ozsoy et al., 2011) ${ }^{[10]}$. In the present study, immunohistochemistry for the proliferation marker Ki67 displayed mild immunopositivity in the nuclei of basal layer of epidermis in fibropapillomas (Beytut, 2017) ${ }^{[1]}$. This was in agreement with the findings of Ozsoy et al. (2011) ${ }^{[10]}$ and Kumar et al. (2013) [7], who observed Ki67 immunoreactivity in the basal layers of the epidermis and connective tissue of the papillomas.

In this study, the authors described clinical and pathological findings of bovine papillomatosis. Immunohistochemical studies like VEGF, EGFR and Ki67 were very useful to detect cellular proliferation in the bovine cutaneous papillomatosis.

\section{References}

1. Beytut E. Pathological and immunohistochemical evaluation of skin and teat papillomas in cattle. Turkish Journal of Veterinary and Animal Sciences. 2017; 41:204-212.
2. Borku MK, Atalay O, Kibar M, Cam Y, Atasever A. Ivermectin is an effective treatment for bovine cutaneous papillomatosis. Research in Veterinary Science. 2007; 83:360-363.

3. Culling CFA. Hand book of Histopathological and Histochemical techniques (Including Museum Techniques). 1974; 3:36.

4. Hamad MA, Shammari AMA, Odisho SM, Yaseen NY. Molecular epidemiology of bovine papillomatosis and identification of three genotypes in central Iraq. Intervirology, 2018, 1-10.

5. Jeremiah OT, Fagbohun OA, Babalola OJ. Molecular detection of bovine papilloma viruses associated with cutaneous warts in some breeds of Nigerian cattle. International Journal of Biotechnology and Biochemistry, 2016; 12(2):123-130.

6. Jelinek F, Tachezy R. Cutaneous papillomatosis in cattle. Journal of Comparative Pathology. 2005; 132:70-81.

7. Kumar P, Nagarajan N, Kumar D, Bind RB, Somvanshi R. Detection and quantification of bovine papillomaviruses (BPVs) in cutaneous warts of cattle and buffaloes. Advances in Animal and Veterinary Sciences. 2013; 1(2):53-58.

8. Martano M, Power K, Restucci B, Pagano I, Altamura G, Borzacchiello G et al. Maiolino P 2018 Expression of vascular endothelial growth factor (VEGF) in equine sarcoid. BMC Veterinary Research. 2013; 14(26):1-9.

9. Mathi PM, Saranya K, Umadevi U, Umakanthan T. An effective treatment regimen forwards in cattle. Journal of Veterinary Science and Animal Husbandry. 2016; 4(2):12.

10. Ozsoy SY, Ozyildiz Z, Guzel M. Clinical pathological and immunohistochemical findings of bovine cutaneous papillomatosis. Ankara Universitesi Veteteriner Fakultesi Dergesi. 2011; 58:161-165.

11. Somvanshi R. Papillomatosis in buffaloes: A less-Known Disease. Transboundary and Emerging Diseases. 2011; 58:327-332.

12. Tozato CC, Lunardi M, Alfieri AF, Otonel RAA, Santis GWD, Alcantara BKD et al. Teat papillomatosis associated with bovine papilloma virus types $6,7,9$, and 10 in dairy cattle from Brazil. Brazilian Journal of Microbiology. 2013; 44(3):905-909.

13. Ugochukwu ICI, Aneke CI, Idoko IS, Sani NA, Amoche AJ, Mshiela WP et al. Bovine papilloma: etiology, pathology, immunology, disease status, diagnosis, control, prevention and treatment: a review. Comparative Clinical Pathology, 2018, 1-9. 